\title{
Review
}

\section{Current Opinion in Pharmacological and Non-Pharmacological Treatment of Migraine: A Focus on Calcitonin Gene-Related Peptide}

\author{
Babar Mirza, MD ${ }^{\prime *}$;Wageha Akel, MD² \\ 'Windsor University School of Medicine, St. Kitts, West Indies \\ ${ }^{2}$ St. James School of Medicine, St.Vincent and the Grenadines, West Indies
}

"Corresponding author

Babar Mirza, MD

Windsor University School of Medicine, St. Kitts,West Indies; E-mail: babarmirza@student.windsor.edu

\section{Article information}

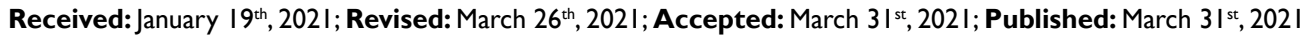

\section{Cite this article}

Mirza B,AkelW. Current opinion in pharmacological and non-pharmacological treatment of migraine:A focus on calcitonin gene-related peptide. Neuro Open J.202I; 8(I): 5-10. doi: 10.17/40/NOJ-8-136

\section{ABSTRACT}

The most common neurologic disorder is migraines. Migraine is defined as throbbing headaches that can be associated with auras. The headaches are episodic and can be debilitating in quality. Migraines can be triggered by emotional stress, lack of sleep, bright lights, loud noise, certain foods, and other environmental factors. The most effective way of preventing migraines is to avoid these triggers. A migraine can begin with prodromes or warning signs such as loss of vision, loss of motor reflexes or sensation. In this review, the types of migraine, signs and symptoms, pathways leading up to auras, and detailed pathophysiology will be discussed. The pathophysiology of a migraine consists of three different mechanisms: 1) cortical spreading depression, 2) the trigemino vascular system, and 3) sensitization. Three different treatment methods for a migraine will be discussed: 1) pharmacological, 2) non-pharmacological and 3) lifestyle modifications. Lifestyle modifications include eating a healthy diet, exercising, and maintaining proper sleep hygiene. Pharmacological treatments can be preventative or abortive. The latest migraine treatment of calcitonin gene-related peptide (CGRP) antagonist use will be discussed in this review and compared to other treatments such as nonsteroidal anti-inflammatory drugs (NSAIDs), anticonvulsants, and Triptans. Future research methods to prevent and better treat migraine headaches are considered a hot topic in medicine and these novel methods will be discussed.
\end{abstract}

\section{Keywords}

Migraine treatment; CGRP antagonists; Neuromodulation; Nerve stimulation; Trigeminal activation;

Cortical spreading depression.

\section{INTRODUCTION}

A migraine is a debilitating headache that is throbbing in quality. Migraine headaches are located unilaterally, but in rare instances they can be bilateral. Migraine headaches affect both children and adults of varying ages. Globally, the third most common illness is migraine headaches affecting $14.7 \%$ of the world population. ${ }^{1}$ Migraine is more common in women than men due to female hormones. Although migraine causes are not fully understood, it has been determined that genetics and environmental factors play a major role. Migraine can be triggered by the following: 1) hormonal changes in women such as changes in estrogen throughout their menstrual cycle, 2) hormonal treatments or oral contraceptives (OCPs) have shown to exacerbate migraine, 3) alcoholic and caffeinated beverages, 4) emotional and physical stress, 5) environmental triggers such as bright lights, loud noises, strong smells, 6) changes in sleep patterns, and 7) lack of healthy diet or consuming high amounts of cheese, salty foods, processed foods and monosodium glutamate (MSG) products. $^{2}$

A migraine headache consist of three events: 1) the migraine prodrome, 2) the aura, and 3) the headache. Migraine prodromes are symptoms that occur a day or 2 before the onset of a migraine. About $77 \%$ of patients complain of prodromes. ${ }^{2}$ The most commonly reported prodromes include yawning, constipation, neck stiffness, slurred speech, and irritability. Approximately $25 \%$ of all migraine patients suffer from the migraine aura. ${ }^{2}$ It is understood that the aura occurs before the headache phase, although some patients may experience the aura during the migraine headache. It is important to point out that auras are not exclusive to migraines, they may be present in other conditions as well. 
The headache phase of a migraine is commonly unilateral and throbbing or pulsating in quality. Patients often experience nausea and vomiting during a migraine attack. Migraine sufferers usually complain of light and sound sensitivity. Most patients tend to lie down in a quiet room in order to prevent a migraine from increasing in intensity. An untreated migraine headache can last up to several days. ${ }^{3}$ Once the throbbing pain ceases, the patient may experience fatigue, exhaustion, and generalized weakness.

The different migraine subtypes include the following: 1) hemiplegic migraine, 2) retinal migraine, 3) chronic migraine, 4) vestibular migraine, and 5) menstrual migraine. ${ }^{4}$

The diagnoses of a migraine is based on the international classification of headaches. ${ }^{4}$ The literature states that a migraine should have at least 5 episodes with the following criteria: 1) The duration of the headache should be between 4 to 72-hours, 2) The head should be unilateral in location or pulsating in quality, caused or aggravated by physical activity, and moderate to severe pain in intensity, and 3) It should be associated with photophobia and phonophobia.

\section{MIGRAINE PATHOPHYSIOLOGY}

There are several studies that suggest that a migraine is a sensory dysmodulation and failure of brainstem processing of sensory stimuli due to poor regulation of vascular tone leading to pain. ${ }^{5} \mathrm{~A}$ study done in 1948 by Wolff Harold suggests that headaches due to excitatory effects are a prerequisite to changes in the neurovascular system and thus are followed by pain. ${ }^{6}$ The brainstem activation may be involved in the trigger phase of migraines. Several neurotransmitters and neuromodulators have been implicated in the pathogenesis of migraine. A few studies have shown that migraine patients have an increased level of calcitonin gene-related peptide (CGRP) in the blood ${ }^{7}$ and/or hypersensitivity on CGRP receptors.

Patients with migraine have increased levels of CGRP, a neuropeptide in the central and peripheral nervous system, which binds to its receptor causing a cascade of events resulting in vasodilation and possibly neurogenic inflammation. The same process peripherally, stimulates the CGRP receptors in the brain stem which is associated with migraine pain. This results in mast cell degranulation and vasodilation and protein extravasation. ${ }^{8}$ CGRP affects the trigeminovascular system hence leading to vasodilation and inflammation and central nervous system (CNS) activation that results in increased transmission of nociception.

There are several classes of medications that have been widely used in the management of migraines which target different mechanisms of migraine pathophysiology.

\section{INTERVENTIONS |}

\section{Nonpharmacologic Interventions}

There are several non-pharmacological methods that have been recommended in the treatment of migraine headaches.
It is important to educate patients on the pathophysiology of the disease in order to avoid triggers, ${ }^{9,10}$ and aid in successful management of the disorder. Furthermore, dietary modifications and lifestyle changes been incorporated in to the management plans of migraines ${ }^{11}$ as it has been shown to have an impact on the overall outlook.

A few studies have shown some promising results, however more research is needed to determine their effectiveness. Biofeedback treatment of migraine shows some efficacy in a recent meta-analysis of pediatric population. ${ }^{12}$ Several institutions have explored body/mind treatment and behavioral modification as an effective means of migraine treatment. ${ }^{13}$ There has been some support in the treatment of chronic headaches with the use of massage therapy ${ }^{14,15}$ and acupuncture of the upper neck and thoracic region; ${ }^{15,12}$ however, the effectiveness of these studies is subjective and requires a more in-depth understanding of the pathophysiological alterations.

\section{Electrical Interventions}

The employment of electrical stimulation through device stimulation has been under investigation for some time. This relieves pain via the "gate theory of pain" by use of neurotransmitter and alteration of $\gamma$-aminobutyric acid (GABA)-ergic systems. ${ }^{5,16}$ The several types of stimulation can be divided into invasive procedures and non-invasive procedures.

There are two types of invasive procedures; occipital nerve stimulation (ONS) and sphenopalatine ganglion stimulation (SGS). ONS uses a subcutaneous pulse generating device which is implanted superficially to the fascia at the level of $\mathrm{C}_{1}{ }^{9}$ One study conducted through 2007-2013 achieved greater than 30\% reduction in moderate to severe headaches days per month. ${ }^{12}$ A second invasive method uses the largest parasympathetic (sphenopalatine) ganglion that exists extracranially. Stimulation of this sphenopalatine ganglion interrupts the parasympathetic outflow and intern modulates the sensory input in the trigeminal nucleus. ${ }^{17}$ Studies are insufficient to prove the efficacy of this modality, however one study of 10 patients showed an alleviation in $50 \%$ of migraines. ${ }^{18}$

There are three types of non-invasive procedures; transcutaneous electrical stimulation of supraorbital nerve (TESoSN), transcranial magnetic stimulation (TMS), and vagus nerve stimulation (VNS), for review see Samsam and Ahangari 2017. TESoSN and TMS use a single electrical pulse that is placed close the skin to transmit transcutaneously through electrodes to the nerves. TESSoSN has some moderate effects, however, it needs to be used on a daily basis for several minutes to alleviate the symptoms of migraine. TMS is used to activate the motor cortex and alleviate the pain of migraine with aura. The stimulation passes through a coil placed on the individual's head. This device can be placed in a target area which depolarizes the area for a short period of time. ${ }^{19}$ In a recent animal study, TMS was able to inhibit chemical and mechanical spreading of $3^{\text {rd }}$ order neurons in the thalamocortical region of the brain. However, it did not have an effect on the $2^{\text {nd }}$ order neurons in the complex. ${ }^{19}$ VNS has been 
widely used in treatment of epileptic seizures refractory to medical intervention and treatment of headaches. This works by changing the blood oxygenation levels and thus altering blood flow to different areas of the brain. ${ }^{14}$ The VNS has also shown changes in the release of neurotransmitter and vasoactive substances. ${ }^{14}$ These modulations take place in the trigeminovascular pain center of the brain. Continuous stimulation of this region has shown to reduce production of glutamate, therefore modulating nociceptors of the craniofacial region. ${ }^{14}$

\section{Pharmacologic Interventions}

Migraine patients can benefit from preventive and abortive treatments. A few classes of drugs that have been studied to help terminate migraines include anti-inflammatory drugs, neurotransmitter modulation, opiate receptor agonists, and calcium channel blockers and others. These are widely being used today by several practitioners as abortive measures more than preventative measures. More recent studies involve the use of CGRP antagonist and the monoclonal antibodies against CGRP or its receptor which have gained some traction in migraine treatment. ${ }^{20}$

Non-steroid anti-inflmmatory: Non-steroid anti-inflammatory drugs (NSAIDs) are some of the most widely used medications for migraine headaches serving as a baseline for treatment. These drugs act on cyclooxygenase enzymes involved in prostaglandin production. Prostaglandins are involved with the inflammatory process and thus cause dilation of vessels, a key component to migraine headaches. By blocking this process, medications such as aspirin, naproxen and meclofenamate, ${ }^{21}$ have proven to be effective management in abortive migraine treatment. Although it has relatively minor side-effects such as stomach ulcer formation and mild hepatic and renal toxicity, it is still widely used for mild migraine headaches.

Triptan: Another family of medication is the Triptan family, Serotonin (5-HT) receptor agonists. 5-HT is a monoamine neurotransmitter which acts as a vasoconstrictor of cerebral vessels. ${ }^{22}$ This plays a significant role in treating migraine as well as strokes and vasospasms. ${ }^{23,24}$ Sumatriptan, a 5-HT agonist primarily works on the trigeminal afferents and nucleus. The inhibition of the peripheral presynaptic trigeminovascular neurons have yielded effective and rapid abortion of migraine headaches. ${ }^{25}$ However, there have been reports of non-localized vasoconstrictive effects leading to dizziness from coronary artery vasoconstriction, and asthenia, and somnolence from impediment of blood flow to different cortical areas. ${ }^{26}$

Glutamate: Glutamate is a major excitatory neurotransmitter of the trigeminal ganglion and some studies have shown that blocking its effects can play a role in migraine treatment. Glutamate allows the influx of calcium ions through presynaptic channels leading to the activation of $\mathrm{N}$-methyl-D-aspartate (NMDA) receptors allowing propagation of migraine through the cortex ${ }^{27}$ By blocking this function, glutamate antagonists effectively decrease excitement of the ganglion and therefore, decrease central terminal activity involved with trigeminal pain processing pathway. ${ }^{28}$ However, glutamate antagonists is a form of non-targeted treatment and the side-effects vary from hallucinations and paranoia to catatonia, anesthesia and ataxia.

Opiate: Opiates work by modulating nociceptors by decreasing calcium influx into the neurons. This ultimately blocks neurotransmitter vesicle docking on the presynaptic cleft and decreases its release. Furthermore, they hyperpolarize the postsynaptic neuron by having potassium efflux and blocking transmission even more. ${ }^{29}$ Opiates block neurotransmission within the trigeminal nuclei and cause neurogenic vasodilation by inhibiting vasoactive substances of the trigeminal vasculature. ${ }^{15}$ However, due to its short-acting effect, wide scale abuse has been observed leading to overuse and drug tolerance, and addiction in chronic users. $^{30,31}$

\section{CALCITONIN GENE RELATED PEPTIDE |}

In the last three decades, the neuropeptide CGRP, has been implicated to play a role in migraine pathogenesis. Migraine patients have been found to have elevated CGRP in their blood and hypersensitive receptor activity in the trigeminal area. CGRP is a local vasodilator causing alterations in blood flow and hyperaemia of its surrounding tissues. Studies have shown migraine sufferers have increased levels of CGRP. ${ }^{25,32}$ Once it binds to its receptor a cascade of events ensues which leads to vasodilation and plasma protein extravasation leading to neurogenic inflammation associated with migraine pain in the periphery. CGRP release centrally in the trigeminal nucleus activated the secondary neurons and the trigeminothalamic pathway to register the pain in higher brain centers. ${ }^{8}$ The CGRP antagonists, called the Gepant family of drugs including the Olcegepant and Telcagepant proved effective in treating migraine headache, ${ }^{20}$ but were discontinued due to side effects and liver toxicity although newer drugs are still being tested. ${ }^{18}$ Nevertheless, CGRP antagonists compared to triptans cause relatively low rebound hypertension attack. ${ }^{21}$

In recent years, another class of drugs called monoclonal antibodies against CGRP were developed and proved to be effective in preventing and aborting migraine headaches. ${ }^{20}$ Although one study with CGRP antagonists, telcagepant, was effective in lowering migraine pain and maintaining a pain-free state for a minimum of two hours at $400 \mathrm{mg},{ }^{33}$ it has shown to have some hepatotoxic effects and these medications are no longer being used. For migraine prevention the following were approved by the Food and Drug Administration (FDA); Erenumab, fremanezumab, and galcanezumab. Fremanezumab (AJOVY) is a monoclonal antibody that selectively targets the CGRP ligand, therefore blocking this cascade of events, and thus preventing activation of the trigeminal system in migraine patients. ${ }^{34}$ One major benefit of using monoclonal antibodies against CGRP is the long-lasting halflife which can range for a few weeks. This allows convenience and decreased injections for patients suffering from chronic recurrent migraines. Furthermore, stimulation of the trigeminal nerve releases CGRP thereby activating caudal neurons and further progressing nociceptor activation. By blocking this pathway, monoclonal antibodies against CGRP inhibit the vasodilatory and nociceptor transmission and ultimately treat migraine pain. The monoclonal antibody approach for modulating CGRP activity, 


\begin{tabular}{|c|c|c|c|c|}
\hline Drug & $\begin{array}{l}\text { Dose, Route of } \\
\text { Administration }\end{array}$ & Side Effects & Effects & Reference \\
\hline $\begin{array}{l}\text { The ALD } 403 \\
\text { (Eptinezum ab by Alder): mAb } \\
\text { against CGRP }\end{array}$ & $\begin{array}{l}100 \mathrm{mg} / 3 \text {-months } \mathrm{OR} \\
300 \mathrm{mg} / 3 \text {-months } \\
\text { Intravenous infusion }\end{array}$ & $\begin{array}{l}\text {-Hypersensitivity reactions including } \\
\text { rash, angioedema, urticaria } \\
\text {-Nasapharyngitis } \\
\text {-Stuffy nose, } \\
\text {-throat irritation }\end{array}$ & $\begin{array}{l}\text { Prevent migraine } \\
\text { heaaches in adults }\end{array}$ & 34 \\
\hline $\begin{array}{l}\text { LY295I } 742 \\
\text { (Gal- canezumab by Eli Lilly): mAb } \\
\text { against CGRP }\end{array}$ & $\begin{array}{l}240 \mathrm{mg} / \mathrm{month} \\
\text { Subcutaneous }\end{array}$ & $\begin{array}{l}\text {-Difficulty breathing } \\
\text {-Hives, itching, skin rash } \\
\text {-Tachycardia } \\
\text {-Fever }\end{array}$ & $\begin{array}{l}\text { Prevent migraine } \\
\text { heaaches in adults }\end{array}$ & 35 \\
\hline $\begin{array}{l}\text { TEV- } 48 \text { I } 25 \\
\text { (Fre-manezum- ab-Ajovy by Teva): } \\
\text { mAb against CGRP. Used for both } \\
\text { chronic and episodic migraine }\end{array}$ & $\begin{array}{l}225 \mathrm{mg} / \text { month OR } \\
675 \mathrm{mg} / 3 \text {-months } \\
\text { Subcutaneous }\end{array}$ & $\begin{array}{l}\text {-Injection site reaction of mild or } \\
\text { moderate severity } \\
\text {-Tachycardia } \\
\text {-Fever } \\
\text {-Hives, itching, skin rash }\end{array}$ & $\begin{array}{l}\text { Treat episodes of cluster } \\
\text { headaches in adults } \\
\text { Prevent migraine } \\
\text { heaaches in adults }\end{array}$ & 36,37 \\
\hline
\end{tabular}

such as Fremanezumab, has shown to have little adverse reactions. In one study, a total of 875 patients ranging between 18-70-years of age were randomized over a 3-month double-blind trial. ${ }^{7}$ The baseline of migraine days per month was 9 across treatment groups. Upon monthly and quarterly dosing, Fremanezumab demonstrated a decrease of almost $50 \%$ of migraine days per month.

Table 1 is a summary of some recent CGRP affecting drugs that have been approved and some are still in clinical trials for the treatment of migraine.

For a recent review of the drugs used in the prevention and treatment of migraine please see Golden GL, 2019.

\section{DISCUSSION}

The purpose of this article was to discuss the pathomechanism behind migraines and briefly discuss the medical treatments being used by physicians today. The use of non-pharmacological management strategies is often used in very mild cases of migraine headaches. Furthermore this methodology is highly subjective, and reserved for susceptible patients to this form of treatment.

Electrical interventions have widely been used for chronic, treatment resistant migraine headaches. These are based on the widely accepted "gate-theory of pain" for treatment. The different methods, both invasive and noninvasive have shown to modulate depolarization and affect the release glutamate in the presynaptic cleft. However, these methods of management often required continuous stimulus along with adjuvant therapy with pharmacological intervention. Furthermore, alterations in the GABA-ergic system can have undesirable side effects of sedation.

Being able to medicate at home meant patients were more likely to adhere to treatment and feel empowered. Several pharmacological interventions have shown to modulate migraine by altering different mechanisms of the associated pathophysiology.

Based on the primitive understanding of migraine pain being associated with increased pressure of the trigeminal vasculature, NSAIDs were the go-to medications. The widespread use of NSAIDs to decrease prostaglandin production and thus inhibit vasodilation was, and has always been the first line of defense for pharmacological therapy in migraine management. However, this mechanism was not localized and was widely used as a baseline for mild migraines.

5-HT has shown to play a role in cerebrovascular vasoconstriction. The Triptan class of medication is used to treat and prevent migraine headaches. 5-HT agonist primarily works on the trigeminal ganglion leading to presynaptic inhibition of trigeminovascular neurons. However, the vasoconstrictive effects of some members of the triptan family have been reported to be non-localized and this have led to dizziness and asthenia. Furthermore, coronary artery vasoconstriction has limited the use of this medication in hypertensive patients, diabetics, and hyperlipidemic patients. Nevertheless, triptans remain one of the first-choice drugs in the treatment of acute migraines. ${ }^{38}$

Glutamate plays a role on the NMDA receptors which allow the propagation of migraine through the cortex. By blocking this excitatory neurotransmitter, glutamate antagonists have demonstrated decreased migraine progression. However non-targeted treatments have led to side effects varying form hallucinations and paranoia to catatonia, anesthesia and ataxia.

Opiates have blocked neurotransmitter release in the trigeminal system which lead to vasodilation, and thus have altered the vasculature causing vasoconstriction. However due to shortacting effect it has led to drug addiction and tolerance. This has a propensity for abuse and has therefore been used as a last resort amongst practitioners.

The most recent studies on the pathomechanism of migraine headaches involves finding CGRP and its role in trigeminal vasculature. CGRP binds to its receptor and a cascade of events ensues leading to neurogenic inflammation associated with migraine pain. This involves mast cell degranulation and vasodilation and protein extravasation. The FDA has approved the use of monoclonal antibodies which target the CGRP ligand and ultimately block it from reacting on its receptor. A benefit to the use of monoclonal antibodies is the long lasting half-life of up to a few weeks. AJOVY has shown promising benefits in its development and trials. It has shown to decrease migraine days 
per month by up to $50 \%$. The convenience and easy adherence to management has demonstrated promising results amongst physician use. Furthermore, there have been relatively little side effects of this medication primarily involved with allergic reaction.

\section{CONCLUSION}

The pathogenesis of migraine headaches, according to recent literature, suggests vasodilation of the trigeminovascular system. This leads to dysregulation of pain and vascular tone in the region propagating downstream trigeminal pain. Several medications have been used to inhibit vasodilation in the region, decrease propagation of electrical stimuli, and inhibit neurotransmitter transmission. A number of studies have shown an increased level of CGRP neuropeptide in migraine patients. CGRP, once bound to its respective receptor, leads to vasodilation, neurogenic inflammation, mast cell degradation, and protein extravasation. CGRP can also activate secondary neurons in the trigeminal nucleus when released from central axons of the primary trigeminal sensory neurons. The recent development of monoclonal antibodies to target CGRP and its receptor is widely becoming the mainstay management for migraine headaches. ${ }^{39}$ Although, there have been many innovations in the past several decades in migraine management, it might be time to consider CGRP as one of the first-line treatment for migraine headaches.

\section{ACKNOWLEDGEMENTS}

We thank Dr. Mohtashem Samsam for his continued support and guidance.

\section{CONFLICTS OF INTEREST}

The authors declare that they have no conflicts of interest.

\section{REFERENCES}

1. Creeney H. CGRP pathway monoclonal antibodies. Web site. https://www.migrainetrust.org/living-with-migraine/treatments/ calcitonin-gene- related-peptide-pathway-monoclonal-antibodies/. Accessed December 4, 2020.

2. Swanson JW, Riggin EA. "Migraine." Mayo Clinic, Mayo Foundation for Medical Education and Research. Web site. www.mayoclinic.org/diseases-conditions/migraine-headache/ symptoms-causes/syc-20360201. Accessed December 4, 2020.

3. Karatas H, Erdener SE, Gursoy-Ozdemir Y, Lule S, Eren-Koçak E, Sen ZD, Dalkara T. Spreading depression triggers headache by activating neuronal panx1 channels. Science. 2013; 339(6123): 10921095. doi: $10.1126 /$ science. 1231897

4. Cutrer M. Pathophysiology of migraine. Semin Neurol. 2006; 26(2): 171-180. doi: 10.1055/s-2006-939917

5. Sprenger T. Goadsby PJ. Migraine pathogenesis and state of pharmacological treatment options. BMC Med. 2009; 16: 71. doi: 10.1186/1741-7015-7-71
6. Silberstein SD. Treatment recommendations for migraine. Nat Clin Pract Neurol. 2008; 4(9): 482-489. doi: 10.1038/ncpneu-ro0861

7. Dodick DW, Silberstein SD, Bigal ME, Yeung PP, Goadsby PJ, Blankenbiller T. Effect of fremanezumab vs placebo on prevention of episodic migraine. JAMA. 2018; 319(19): 1999-2008. doi: 10.1001/jama.2018.4853

8. Samsam M. Central nervous system acting drugs in treatment of migraine headache. Cent Nerv Syst Agents Med Chem. 2012; 12: 158172. doi: $10.2174 / 187152412802430147$

9. Saper JR, Dodick DW, Silberstein SD, McCarville S, Sun M, Goadsby PJ, ONSTIM Investigators. Occipital nerve stimulation for the treatment of intractable chronic migraine headache: ONSTIM feasibility study. Cephalalgia. 2011; 3 1(3): 271-285 . doi : $10.1177 / 0333102410381142$

10. Stanton-Hicks M, Salamon J. Stimulation of the central and peripheral nervous system for the control of pain. J Clin Neurophysiol. 1997; 14(1): 46-62. doi: 10.1097/00004691-199701000-00004

11. Bhola R, Kinsella E, Giffin N, Lipscombe S, Ahmed F, Weatherall $\mathrm{M}$, et al. Single-pulsetranscranial magnetic stimulation (sTMS) for the acute treatment of migraine: Evaluation of outcome data for the UK post market pilot program. $J$ Headache Pain. 2015; 16: 535. doi: 10.1186/ s10194-015-0535-3

12. Stubberud A, Varkey E, McCrory DC, Pedersen SA, Linde M. Biofeedback as Prophylaxis for Pediatric Migraine: A Metaanalysis. Pediatrics. 2016; 138(2): pii: e20160675. doi: 10.1542/ peds.2016-0675

13. Wells RE, Loder E. Mind/Body and behavioral treatments: The evidence and approach. Headache. 2012; 52 Suppl 2: 70-75. doi: 10.1111/j.1526-4610.2012.02238.x

14. Sun Y, Gan TJ. Acupuncture for the management of chronic headache: A systematic review. Anesth Analg. 2008; 107(6): 20382047. doi: 10.1213/ane.0b013e318187c76a

15. Henry TR, Bakay RA, Votaw JR, Pennell PB, Epstein CM, Faber TL, et al. Brain blood flow alterations induced by therapeutic vagus nerve stimulation in partial epilepsy: I. Acute effects at high and low levels of stimulation. Epilepsia. 1998; 39(9): 983-990. doi: 10.1111/j.1528-1157.1998.tb01448.x

16. Stojanovic MP. Stimulation methods for neuropathic pain control. Curr Pain Headache Rep. 2001; 5(2): 130-137. doi: $10.1007 /$ s11916-001-0081-3

17. Rhee TG, Harris IM. Gender differences in the use of complementary and alternative medicine and their association with moderate mental distress in U.S. adults with migraines/severe headaches. Headache. 2017; 57(1): 97-108. doi: 10.1111/head.12986

18. Tepper SJ, Cleves C. Telcagepant, a calcitoningene related peptide antagonist for the treatment of migraine. Curr Opin Investig 
Drugs. 2009; 10: 711-720.

19. Andreou AP, Holland PR, Akerman S, Summ O, Fredrick J, Goadsby PJ. Transcranial magnetic stimulation and potential cortical and trigeminothalamic mechanisms in migraine. Brain. 2016; 139(Pt 7): 2002-2014. doi: 10.1093/brain/aww118

20. Samsam M., Targeting calcitonin gene related peptide and its receptor by monocolonal antibody, new developments in the treatment of migraine. Neuro Open J. 2015; 2: e6-e10. doi.: 10.17140/NOJ-2-e004

21. Gokani T. Ayurveda--the science of healing. Headache. 2014; 54(6): 1103-1106. doi: 10.1111/head.12363

22. McBean DE, Sharkey J, Ritchie IM, Kelly PA. Evidence for a possible role for serotonergic systems in the control of cerebral blood flow. Brain Res. 1990; 537: 307-310. doi: 10.1016/00068993(90)90374-K

23. Merison K, Jacobs H. Diagnosis and treatment of childhood migraine. Curr Treat Options Neurol. 2016; 18(11): 48. doi: 10.1007/ s11940-016-0431-4

24. Happe S, Peikert A, Siegert R, Evers S. The efficacy of lymphatic drainage and traditional massage in the prophylaxis of migraine: A randomized, controlled parallel group study. Neurol Sci. 2016; 37(10): 1627-1632. doi: 10.1007/s10072-016-2645-3

25. Foroughipour M, Golchian AR, Kalhor M, Akhlaghi S, Farzadfard MT, Azizi H. A sham-controlled trial of acupuncture as an adjunct in migraine prophylaxis. Acupunct Med. 2014; 32(1): 12-16. doi: 10.1136/acupmed-2013-010362

26. Ramadan NM, Skljarevski V, Phebus LA, Johnson KW. 5-HT1F receptor agonists in acute migraine treatment: a hypothesis. Cephalalgia. 2003; 23: 776-785. doi: 10.1046/j.14682982.2003.00525.x

27. Tottene A, Urbani A, Pietrobon D. Role of different voltagegated $\mathrm{Ca} 2+$ channels in cortical spreading depression: specific requirement of $\mathrm{P} / \mathrm{Q}$-type Ca2+ channels. Channels (Austin). 2011; 5: 110-114. doi: 10.4161/chan.5.2.14149

28. Knyihár-Csillik E, Tajti J, Samsam M, Sáry G, Buzás P, Vécsei L. Effect of a serotonin agonist (Sumatriptan) on the peptidergic innervation of the rat cerebral dura mater and on the expression of c-fos in the caudal trigeminal nucleus in an experimental migraine model. J Neurosci Res. 1997; 48: 449-464.

29. Matharu MS, Bartsch T, Ward N, Frackwiak RS, Weiner R, Goadsby PJ. Central neuromodulation in chronic migraine patients with suboccipital stimulators: A PET study. Brain. 2004; 127: 220
230. doi: $10.1093 /$ brain/awh022

30. Bahra A, Walsh M, Menon S, Goadsby PJ. Does chronic daily headache arise de novo in association with regular use of analgesics? Headache. 2003; 43: 179-190. doi: 10.1046/j.15264610.2003.03041.x

31. Radat F, Creac'h C, Guegan-Massardier E, Mick G, Guy N, Fabre N, et al. Behavioral dependence in patients with medication overuse headache: A cross-sectional study in consulting patients using the DSM-IV criteria. Headache. 2008; 48: 1026-1036. doi: 10.1111/j.1526-4610.2007.00999.x

32. Gallai V, Sarchielli P, Floridi A, Franceschini M, Codini M, Glioti $G$, et al. Vasoactive peptide levels in the plasma of young migraine patients with and without aura assessed both interictally and ictally. Cephalalgia. 1995; 15(5): 384-390. doi: 10.1046/j.14682982.1995.1505384.x

33. Robbins L. New Migraine Treatments: Oral Gepants, Ditan Tablet, and More. Web site. https://www.practicalpainmanagement. $\mathrm{com} /$ treatments / pharmacological/new-migraine-medicationsoral-gepants-ditan-tablet-more. May 14, 2020.

34. Bigal ME, Walter S, Rapoport AM. Therapeutic antibodies against CGRP or its receptor. Br J Clin Pharmacol. 2015; 79(6): 886895. doi: $10.1111 /$ bcp. 12591

35. Bangs ME, Kudrow D, Wang S, Oakes TM, Terwindt GM, Magis $\mathrm{D}$, et al. Safety and tolerability of monthly galcanezumab injections in patients with migraine: integrated results from migraine clinical studies. BMC Neurology. 2020; 20: 25. doi: 10.1186/s12883-0201609-7

36. Silberstein SD, Dodick DW, Bigal ME, Yeung PP, Goadsby PJ, Blankenbiller T, et al. Fremanezumab for the preventive treatment of chronic migraine. N Engl J Med. 2017; 377: 2113-2122. doi: 10.1056/NEJMoa1709038

37. Dodick DW, Silberstein SD, Bigal ME, Yeung PP, Goadsby PJ, Blankenbiller T, et al. Effect of fremanezumab compared with placebo for prevention of episodic migraine. JAMA. 2018; 319(19): 5-14. doi: 10.1001/jama.2018.4853

38. Evers S, Afra J, Frese A, Goadsby PJ, Linde M, May A, et al. EFNS guideline on the drug treatment of migraine-revised report of an EFNS task force. Eur. J Neurol. 2009; 16: 968-981. doi: 10.1111/j. 14681331.2009.02748.x

39. Spindler BL, Ryan M. Medications approved for preventing migraine headaches. Am J Med. 2020; 133: 664-667. doi: 10.1016/j. amjmed.2020.01.031 